\title{
Verwechslungsgefahr von Prava mit Pravastatinpräparaten
}

Swissmedic

Korrespondenz:

Swissmedic

Hallerstrasse 7

Postfach

CH-3000 Bern 9

www.swissmedic.ch
Betrifft Prava, Kapseln (Lomustin, Zytostatikum) und die folgenden Pravastatingenerika/CoMarketing-Arzneimittel (HMG-CoA-Reduktasehemmer; Statine):

- Pravastatin Nycomed, Tabletten;

- Pravalotin, Tabletten;

- Pravastatin Sandoz, Tabletten;

- Pravatin, Tabletten;

- Pravasta eco, Tabletten;

- Pravastatin Helvepharm, Tabletten;

- Pravastatin Streuli, Tabletten;

- Pravastatin-Teva, Tabletten;

- Pravastax, Tabletten.

Der Swissmedic wurden Fälle von Verwechslungen bzw. Beinaheverwechslungen bei der Verschreibung von Pravastatinpräparaten (HMGCoA-Reduktasehemmer zur Cholesterinsenkung) mit dem Zytostatikum Prava (Lomustin) gemeldet.

Die Zulassungsinhaberin von Prava, BristolMyers Squibb, wird aus diesem Grund eine Namensänderung für ihr Präparat vornehmen und hat die nötigen Schritte bereits eingeleitet.
Es sind aber zum aktuellen Zeitpunkt noch Packungen mit dem Namen Prava im Handel.

Swissmedic bittet alle Ärztinnen und Ärzte, die Präparatenamen und die Dosierungsempfehlung der Pravastatinpräparate vollständig und deutlich auf den Rezepten auszuschreiben.

Gleichzeitig sind alle Apothekerinnen und Apotheker gebeten, alle Verschreibungen von Prava dahingehend zu überprüfen, ob wirklich das Zytostatikum gemeint ist.

Die üblichen Dosierungsempfehlungen des Zytostatikums Prava und der Pravastatinpräparate unterscheiden sich erheblich (Prava: 100$130 \mathrm{mg} / \mathrm{m}^{2}$ Körperoberfläche als Einzeldosis alle 6-8 Wochen; Pravastatinpräparate: 10-40 mg 1mal täglich).

Swissmedic macht darauf aufmerksam, dass die obenerwähnten Vorsichtsmassnahmen (genaue Überprüfung von Indikation und Dosierung) grundsätzlich bei jeder Verschreibung eines Medikamentes beachtet werden müssen. Zusätzlich sollen die Patientinnen und Patienten immer zum Lesen der Packungsbeilage ermuntert werden. 